burden of $1.7(\mathrm{SD} \pm 0.8), 57 \mathrm{Gl}$ reactions $(9.8 \%, 41 \mathrm{pts})$ with an average burden of $2.9(\mathrm{SD} \pm 1.1), 102 \mathrm{SK}$ reactions $(17.5 \%, 64 \mathrm{pts})$ with an average burden of $2.9(\mathrm{SD} \pm 1.1)$ and $109 \mathrm{INFs}(18.7 \%, 79 \mathrm{pts})$ with an average burden of $3.3(\mathrm{SD} \pm 1.0)$. Prevalence of $\mathrm{INJ}$ and $\mathrm{Gl}$ reactions was significantly higher among ETN users than among ADA users (INJ: 76 ADRs/265 ETN users (28.7\%) vs 30 ADRs/196 ADA users (15.3\%), $\mathrm{P}=0.007$; Gl: $30 / 265(11.3 \%)$ vs $9 / 196(4.6 \%), P=0.01)$. The prevalence of INFs and SK reactions did not differ. The perceived burden of Gl reactions was higher for ETN than for ADA users (3.1 \pm 1.0 vs $2.0 \pm 0.7$, $\mathrm{p}=0.006$ ) but did not differ for other clustered ADRs. The number of patients using other bDMARDs was too low for further analysis. Prevalence of bDMARD-induced headache was $1.9 \%$ (12 ADRs/583 pts) with an average burden of $3.4(\mathrm{SD} \pm 0.9)$. Prevalence of bDMARD-induced fatigue was $4.3 \%$ (25 ADRs) with an average burden of $2.7(\mathrm{SD} \pm 1.0)$. Conclusion: Almost half of the RA patients reported bDMARD-induced ADRs. From the patients' perspective INJ reactions have the highest prevalence with a relatively low burden, whereas INFs and headache are less prevalent but give the highest burden of the analysed ADRs. Further studies are required to obtain more insight into the perceived differences in ADRs between bDMARDs.

Disclosure of Interests: Jette van Lint: None declared, Naomi Jessurun: None declared, Sander W. Tas: None declared, Harald Vonkeman: None declared, Bart van den Bemt Grant/research support from: UCB, Pfizer, Abbvie; Speakers bureau: Pfizer, AbbVie, UCB, Biogen, Sandoz, Consultant for: UCB, Novartis and Pfizer, Astrid van Tubergen: None declared, Michael Nurmohamed Grant/research support from: AbbVie, Bristol-Myers Squibb, Celgene, Eli Lilly, Janssen, Menarini, MSD, Mundipharma, Pfizer, Roche, Sanofi and UCB, Consultant for: AbbVie, Bristol-Myers Squibb, Celgene, Eli Lilly, Janssen, Menarini, MSD, Mundipharma, Pfizer, Roche, Sanofi and UCB, Speakers bureau: AbbVie, Bristol-Myers Squibb, Celgene, Eli Lilly, Janssen, Menarini, MSD, Mundipharma, Pfizer, Roche, Sanofi and UCB, Eugène van Puijenbroek: None declared DOI: 10.1136/annrheumdis-2019-eular.5330

\section{FRI0098 SWITCHING RATE OF BIOLOGICAL DMARDS IN RHEUMATOID ARTHRITIS PATIENTS: TREASURE - REAL LIFE DATA}

Umut Kalyoncu, Ali İhsan Ertenli, Abdulsamet Erden, Orhan Küçükşahin, Timuçin Kaşifoğlu, Ediz Dalkılıç, Cemal Bes, Nilüfer Alpay Kanıtez, Hakan Emmungil, Pamir Atagündüz, Belkıs Nihan Coşkun, Burcu Yağız, Süleyman Serdar Koca, Muhammet Çınar, Așkın Ateş, Servet Akar, Onay Gercik, Duygu Ersözlü, Veli Yazısız, Gezmiş Kimyon, Müge Aydın, Rıdvan Mercan, Burak Öz, Nazife Sule Yasar Bilge, Zeynel Abidin Akar, Omer Karadag, Ayse Bahar Kelesoglu Dincer, Sedat Yilmaz, Ufuk ilgen, Yavuz Pehlivan, Ender Terzioğlu, Levent Kılıç, Şükran Erten, Sedat Kiraz. On Behalf of TReasure biological registry, Ankara, Turkey

Background: In rheumatoid arthritis (RA), biologic DMARDs are important treatment options in resistant patients. Inefficacy or side effects may cause switching between these drugs.

Objectives: This study aimed to determine features of patients switching from one biologic DMARD to another in RA treatment and to investigate associated reasons for switching.

Methods: This multicenter, prospective observational cohort study used the TReasure database in which web-based registration of RA and spondyloarthritis patients are being performed in 15 centers across different regions of Turkey. In this study, data of RA patients switching from one biologic agent to another were analyzed. Demographic and clinical data, follow-up duration, time to switch, and reasons for switching were retrieved from the database.

Results: Of the included 2115 RA patients, 829 (39.2\%) switched between biologic agents (switched group) and $1286(60.8 \%)$ continued to receive their current therapies (continued group). The median follow-up duration of all patients was 3.7 years and the median time to switch was 1.1 years. In the switched group, the proportion of females and the median HAQ-DI score were higher as well as disease duration was longer (Table 1). Among the biologic agents used at first, $60.9 \%$ of the patients were receiving an anti-TNF agent and $39.1 \%$ of the patients were receiving other biologic agents (Table 2, figure 1). In the switched group $(n=829)$, the main reasons for switching were secondary inefficacy $(n=269)$, primary inefficacy $(n=238)$, and side effects $(n=178)$ followed by primary or secondary unknown inefficacy $(n=30)$, patient's demand $(n=21)$, physician's request $(n=16)$, willing to be pregnant $(n=7)$, other $(n=31)$, and unknown $(n=54)$.
Table 1. Demographic and clinical features of the study groups

\begin{tabular}{|c|c|c|c|c|c|}
\hline & $\mathbf{N}$ & $\begin{array}{l}\text { Switched } \\
\text { Group }\end{array}$ & $\mathbf{N}$ & $\begin{array}{l}\text { Continued } \\
\text { Group }\end{array}$ & $\mathbf{p}$ \\
\hline $\begin{array}{l}\text { Age, year, median (min- } \\
\text { max) }\end{array}$ & 829 & $54(19-84)$ & 1286 & $55(18-91)$ & 0.121 \\
\hline \multirow{2}{*}{ Sex, $n(\%)$} & & $688(83.0)$ & & $1000(77.8)$ & \multirow{2}{*}{0.003} \\
\hline & & $141(17.0)$ & & $286(22.2)$ & \\
\hline $\begin{array}{l}\text { Body mass index, median } \\
\text { ( } \min -\max )\end{array}$ & 823 & $\begin{array}{c}27.74(14.33- \\
66.1)\end{array}$ & 1256 & $\begin{array}{c}28.05(15.59- \\
58)\end{array}$ & 0.640 \\
\hline $\begin{array}{l}\text { Disease duration, year, } \\
\text { median (min-max) }\end{array}$ & 806 & $12(0-43)$ & 1245 & $8(0-41)$ & $<0.001$ \\
\hline $\begin{array}{l}\mathrm{ESR}, \mathrm{mm} / \mathrm{h} \text {, median (min- } \\
\mathrm{max} \text { ) }\end{array}$ & 538 & $34(2-120)$ & 1029 & $33(2-174)$ & 0.311 \\
\hline $\begin{array}{l}\text { CRP, mg/dL, median } \\
\text { (min-max) }\end{array}$ & 533 & $15.7(0.04-335)$ & 1019 & $14.7(0-11062)$ & 0.859 \\
\hline RF positivity, $\mathrm{n}(\%)$ & 776 & $508(65.5)$ & 1193 & $785(65.8)$ & 0.878 \\
\hline Anti-CCP positivity, $n(\%)$ & 585 & $345(59.0)$ & 945 & $582(61.6)$ & 0.309 \\
\hline $\begin{array}{l}\text { Swollen joint count, } \\
\text { median (min-max) }\end{array}$ & 250 & $3(0-24)$ & 612 & $3(0-32)$ & 0.994 \\
\hline $\begin{array}{l}\text { Tender joint count, } \\
\text { median (min-max) }\end{array}$ & 253 & $6(0-30)$ & 609 & $7(0-44)$ & 0.217 \\
\hline $\begin{array}{l}\text { PtGA-VAS, median (min- } \\
\text { max) }\end{array}$ & 432 & $70(0-100)$ & 954 & $70(0-100)$ & 0.347 \\
\hline $\begin{array}{l}\text { Pain-VAS, median (min- } \\
\text { max) }\end{array}$ & 345 & $70(0-100)$ & 879 & $70(0-100)$ & 0.142 \\
\hline $\begin{array}{l}\text { Fatigue-VAS, median } \\
\text { (min-max) }\end{array}$ & 337 & $70(0-100)$ & 873 & $70(0-100)$ & 0.066 \\
\hline $\begin{array}{l}\text { HAQ-DI score, median } \\
\text { (min-max) }\end{array}$ & 300 & $1(0-3)$ & 832 & $0.9(0-2.95)$ & 0.040 \\
\hline $\begin{array}{l}\text { CDAl score, median (min- } \\
\max \text { ) }\end{array}$ & 115 & $15.5(0-72)$ & 293 & $21.5(0-89)$ & 0.117 \\
\hline $\begin{array}{l}\text { SDAl score, median (min- } \\
\text { max) }\end{array}$ & 113 & $37.5(1.72-137)$ & 290 & $43(2-389)$ & 0.057 \\
\hline
\end{tabular}

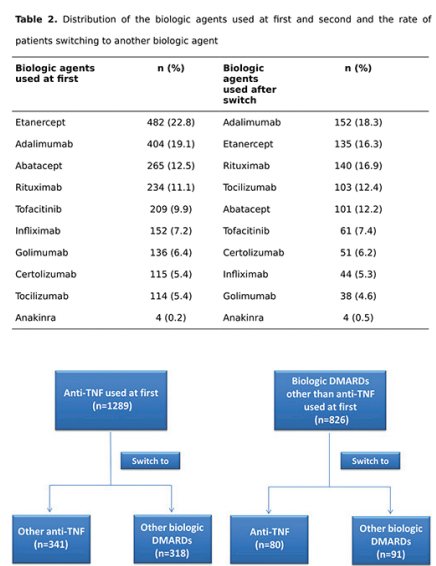

Figure 1. Switching pattern of the switched group

Conclusion: The patients in the Treasure database were followed-up approximately 4 years and about one-third of the patients had to switch from one biologic DMARD to another. The main reasons for this switching were primary $(29.2 \%)$ and secondary $(33.0 \%)$ inefficacy and $20 \%$ of the patients had to switch due to side effects. According to the switching pattern, about half of the patients using an anti-TNF agent at first switched to another anti-TNF agent and the other half switched to other biologic agents.

Disclosure of Interests:

Umut Kalyoncu Grant/research support from: MSD, Roche, UCB, Novartis and Pfizer, Consultant for: MSD, Abbvie, Roche, UCB, Novartis, Pfizer and Abdi Ibrahim, Speakers bureau: MSD, Abbvie, Roche, UCB, Novartis, Pfizer and Abdi Ibrahim, Ali İhsan Ertenli: None declared, Abdulsamet Erden: None declared, Orhan Küçükşahin: None declared, Timuçin Kaşifoğlu: None declared, Ediz Dalkılıç Grant/research support from: MSD and Abbvie, Consultant for: MSD, Abbvie,Roche, UCB, Pfizer and Novartis, Speakers bureau: MSD, Abbvie,Roche, UCB, Pfizer and Novartis, Cemal Bes: None declared, Nilüfer Alpay Kanıtez: None declared, Hakan Emmungil Grant/research support from: MSD, Roche, Pfizer, Abbvie, Consultant for: Novartis, Roche, Speakers bureau: MSD, Roche, Pfizer, Abbvie,Celltrion, Novartis, Pamir Atagündüz: None declared, Belkıs Nihan Coşkun: None declared, Burcu Yağız: None declared, Süleyman Serdar Koca: None declared, Muhammet Çınar: None declared, Aşkın Ateş: None declared, Servet Akar Grant/research support from: MSD, Abbvie, 
Roche, UCB, Novartis, Pfizer, Amgen, Consultant for: MSD, Abbvie, Roche, UCB, Novartis, Pfizer, Amgen, Speakers bureau: Pfizer, Onay Gercik: None declared, Duygu Ersözlü: None declared, Veli Yazısız: None declared, Gezmiş Kimyon: None declared, Müge Aydın: None declared, Rıdvan Mercan: None declared, Burak Öz: None declared, Nazife Sule Yasar Bilge: None declared, Zeynel Abidin Akar: None declared, Omer Karadag: None declared, Ayse Bahar Kelesoglu Dincer: None declared, Sedat Yilmaz: None declared, Ufuk Illgen: None declared, Yavuz Pehlivan: None declared, Ender Terzioğlu: None declared, Levent Kılıç: None declared, Şükran Erten: None declared, Sedat Kiraz: None declared DOI: 10.1136/annrheumdis-2019-eular.5279

\section{FRI0099 PREDICTIVE FACTORS FOR REMISSION ACHIEVEMENT BY TOCILIZUMAB MONOTHERAPY IN PATIENTS WITH RHEUMATOID ARTHRITIS AFTER INADEQUATE RESPONSE TO METHOTREXATE: A POST HOC ANALYSIS OF THE SURPRISE STUDY}

Masaru Kato ${ }^{1}$, Yuko Kaneko², Yoshiya Tanaka ${ }^{3}$, Masayuki Inoo ${ }^{4}$, Hitomi Kobayashi-Haraoka ${ }^{5}$, Koichi Amano ${ }^{6}$, Masayuki Miyata ${ }^{7}$, Yohko Murakawa ${ }^{8}$, Hidekata Yasuoka ${ }^{2}$, Shintaro Hirata ${ }^{9}$, Hayato Nagasawa ${ }^{6}$, Eiichi Tanaka ${ }^{10}$, Nobuyuki Miyasaka ${ }^{11}$, Hisashi Yamanaka ${ }^{10}$, Kazuhiko Yamamoto ${ }^{12}$, Isao Yokota ${ }^{13}$, Tatsuya Atsumi ${ }^{1}$, Tsutomu Takeuchi ${ }^{2}$. ${ }^{1}$ Hokkaido University, Department of Rheumatology, Endocrinology and Nephrology, Sapporo, Japan; ${ }^{2}$ Keio University School of Medicine, Tokyo, Japan; ${ }^{3}$ University of Occupational and Environmental Health, Kitakyushu, Japan; ${ }^{4}$ Utazu Hospital, Ehime, Japan; ${ }^{5}$ Nihon University School of Medicine, Tokyo, Japan; ${ }^{6}$ Saitama Medical University, Kawagoe, Japan; ${ }^{7}$ Fukushima Red Cross Hospital, Fukushima, Japan; ${ }^{8}$ Shimane University Faculty of Medicine, Izumo, Japan; ${ }^{9}$ Hiroshima University Hospital, Hiroshima, Japan; ${ }^{10}$ Tokyo Women's Medical University, Tokyo, Japan; ${ }^{11}$ Tokyo Medical and Dental University, Tokyo, Japan; ${ }^{12}$ The University of Tokyo, Tokyo, Japan; ${ }^{13}$ Hokkaido University, Department of Biostatistics, Sapporo, Japan

Background: Interleukin (IL)-6 pathway inhibitors are suggested by European League Against Rheumatism recommendations to have some advantages in rheumatoid arthritis (RA) patients who are not suitable to use methotrexate as comedication compared with other biological disease-modifying antirheumatic drugs. However, it remains to be elucidated what predicts remission achievement by IL- 6 pathway inhibitor monotherapy.

Objectives: To identify predictive factors for clinical and structural remission by tocilizumab without methotrexate in patients with RA.

Methods: This is a post hoc analysis of the SURPRISE study, a 2-year randomized, controlled study comparing the efficacy of tocilizumab with (ADD-ON) and without methotrexate (SWITCH) in active RA patients despite methotrexate therapy. The primary endpoint was the DAS28-ESR remission $(<2.6)$ at week 24 . The change in modified total Sharp score from baseline to week 52 ( $\Delta$ mTSS/year) was also assessed as an endpoint. The effect of clinical parameters, including serum levels of CRP, IL-6, serum amyloid A (SAA), MMP-3, RF and IgG at baseline, on the achievement of DAS28 remission at week 24 was estimated by logistic regression analysis. Multivariate analysis was performed after adjusting for age, gender, height, body weight, body mass index, disease duration, the dose of glucocorticoids, and that of methotrexate.

Results: In SWITCH ( $\mathrm{n}=96)$, CRP, SAA, RF and DAS28 at baseline showed predictive value for DAS28 remission at week 24 in univariate analysis. Multivariate analysis confirmed SAA, RF and DAS28 at baseline, but not CRP, as predictive factors, with SAA having the highest value (OR $[95 \% \mathrm{Cl}$ ] by decrease of $5.0 \mu \mathrm{g} / \mathrm{mL}=1.011$ [1.002-1.020], $\mathrm{p}=0.01$, ROC-AUC $=0.731$ ). SAA of $<50.0 \mu \mathrm{g} / \mathrm{mL}$ showed extremely high predictive value for DAS28 remission $(\mathrm{OR}[95 \% \mathrm{Cl}]=6.012[1.997-18.096], \mathrm{p}=$ 0.0008 , ROC-AUC $=0.761$ ). Furthermore, structural remission ( $\triangle \mathrm{mTSS}$ / year $\leq 0.5)$ rate at week 52 was significantly higher in patients with SAA of $<50.0 \mu \mathrm{g} / \mathrm{mL}$ than those with SAA of $\geq 50.0 \mu \mathrm{g} / \mathrm{mL}(71 \%$ vs $51 \%$, p $=0.048)$. In contrast, in ADD-ON ( $n=98)$, only DAS28 at baseline showed predictive value for DAS28 remission at week 24 in both univariate and multivariate analysis. In patients with SAA $<50.0 \mu \mathrm{g} / \mathrm{mL}$, both DAS28 remission $(75 \%$ vs $77 \%, p=0.79)$ and structural remission $(71 \%$ vs $68 \%, p=0.71$ ) rate were comparable between SWITCH and ADDON.

Conclusion: SAA levels at baseline can predict the necessity of concomitant methotrexate in tocilizumab initiation in patients with RA. Patients with low levels of SAA at baseline may benefit similarly from tocilizumab therapy with and without methotrexate in terms of achieving clinical and structural remission.

\section{REFERENCE:}

[1] Kaneko Y, et al. Ann Rheum Dis. 2016 Nov;75(11):1917-1923. Kaneko Y, et al. Ann Rheum Dis. 2018 Sep;77(9):1268-1275.

Disclosure of Interests: : Masaru Kato Grant/research support from: GSK, Actelion, Speakers bureau: GSK, Actelion, Bayer, Nippon Shinyaku, Eli Lilly, Chugai, Pfizer, Ayumi, Eisai, Asahi Kasei, Yuko Kaneko Grant research support from: Abbvie, Eisai, Speakers bureau: AbbVie, Astellas, Ayumi, Bristol-Myers Squibb, Chugai, Eisai, Eli Lilly, Jansen, Kissei, Pfizer, Sanofi, Takeda, Tanabe-Mitsubishi, UCB, Yoshiya Tanaka Grant research support from: Abbvie, Astellas, Bristol-Myers Squibb, Chugai, Daiichi-Sankyo, Eisai, Mitsubishi-Tanabe, MSD, Ono, Taisho-Toyama, Takeda, Speakers bureau: Abbvie, Asahi-kasei, Astellas, Bristol-Myers Squibb, Chugai, Daiichi-Sankyo, Eli Lilly, Eisai, Glaxo-Smithkline, Janssen, Mitsubishi-Tanabe, Novartis, Pfizer Japan Inc, Sanofi, Takeda, UCB, YL Biologics, Masayuki Inoo: None declared, Hitomi Kobayashi-Haraoka: None declared, Koichi Amano Grant/research support from: Asahi Kasei Pharma, Chugai Pharmaceutical Co. Ltd., Speakers bureau: AbbVie GK, Eli Lilly Japan K.K., Mitsubishi Tanabe Pharma, Pfizer Japan Inc., Masayuki Miyata: None declared, Yohko Murakawa Grant/research support from: Asahi Kasei Pharmaceutical Co., Ltd., Chugai Pharmaceutical Co., Ltd., Ono Pharmaceutical Co., Ltd., Daiichi Sankyo Co., Ltd., Teijin Pharma Ltd., Eisai Co., Ltd., Nippon Kayaku Co., Ltd., Mitsubishi Tanabe Pharma Corporation, Paid instructor for: Kissei Pharmaceutical Co., Ltd. Janssen Pharmaceutical K.K. Eisai Co., Ltd., Speakers bureau: Ono Pharmaceutical Co., Ltd., Mitsubishi Tanabe Pharma Corporation, Astellas Pharma Inc., UCB Japan Co. Ltd., Chugai Pharmaceutical Co., Ltd., AbbVie GK, Daiichi Sankyo Co., Ltd., AYUMI Pharmaceutical Corporation, Janssen Pharmaceutical K.K., Takeda Pharmaceutical Company Limited, Sanofi K.K. Teijin Pharma Ltd., Eli Lilly Japan K.K., Eisai Co., Ltd., Hidekata Yasuoka: None declared, Shintaro Hirata Grant/research support from: Eli Lilly, UCB, Consultant for: Bristol-Myers Squibb, Jansen, UCB, Paid instructor for: AbbVie, Eisai, Tanabe-Mitsubishi, Speakers bureau: AbbVie, Astellas, Ayumi, Bristol-Myers Squibb, Chugai, Eisai, Eli Lilly, Jansen, Kissei, Pfizer, Sanofi, Takeda, Tanabe-Mitsubishi, UCB, Hayato Nagasawa: None declared, Eiichi Tanaka Speakers bureau: Abbvie, Asah Kasei pharma co., Bristol Myers Squibb, Chugai Pharmaceutical, Daiichi Sankyo Co., Eisai Pharmaceutical, Janssen Pharmaceutical K.K., Nippon Kayaku, Pfizer, Takeda Pharmaceutical, Taisho Toyama Pharmaceutical Co., and UCB Pharma., Nobuyuki Miyasaka: None declared, Hisashi Yamanaka Grant/research support from: AbbVie, Eisai, Bristol-Meyers, Novartis, Behringer, Astellas, Kaken, Nippon-Shinyaku, Pfizer, UCB, Ayumi, Ono, Daiichi-Sankyo, Taisyo-Toyama, Takeda, Tanabe-Mitsubishi, Chugai, Teijin Pharma, Torii, YLbio, Speakers bureau: Bristol-Meyers, Astellas, Pfizer, Daiichi-Sankyo, Takeda, Tanabe-Mitsubishi, Chugai, Teijin Pharma, YLbio, Kazuhiko Yamamoto Grant/research support from: Astellas, BMS, Daiichi-Sankyo, Mitsubishi Tanabe, Pfizer, Ayumi, Takeda, Chugai, Eisai, Taisho Toyama, UCB, Janssen, Eli Lilly, and NIPPON KAYAKU., Speakers bureau: Astellas, BMS, Daiichi-Sankyo, Mitsubishi Tanabe, Pfizer, Ayumi, Takeda, Chugai, Eisai, Taisho Toyama, UCB, Janssen, Eli Lilly, and NIPPON KAYAKU., Isao Yokota Speakers bureau: Chugai Pharma, Tatsuya Atsumi Grant/research support from: Astellas Pharma Inc., Takeda Pharmaceutical Co., Ltd., Mitsubishi Tanabe Pharma Co., Chugai Pharmaceutical Co., Ltd., Daiichi Sankyo Co. Ltd., Otsuka Pharmaceutical Co., Ltd. and Pfizer Inc.. Alexion Inc., Bayer Yakuhin, Ltd,Otsuka Pharmaceutical Co., Ltd. Chugai Pharmaceutical Co., Ltd. Takeda Pharmaceutical Co., Ltd., Eisai Co., Ltd., Bristol-Myers Squibb Co., Daiichi Sankyo Co. Ltd., Mitsubishi Tanabe Pharma Co., Asahi Kase Pharma Co., Consultant for: ONO PHARMACEUTICAL CO., LTD Sanofi K.K. Daiichi Sankyo Co., Ltd. Pfizer Inc., Speakers bureau: Mitsubishi Tanabe Pharma Co., Chugai Pharmaceutical Co., Ltd., Astellas Pharma Inc., Takeda Pharmaceutical Co., Ltd., Pfizer Inc.,Daiichi Sankyo Co. Ltd., Bristol-Myers Squibb Co., Eli Lilly Japan K.K., Tsutomu Takeuchi Grant/ research support from: Astellas Pharma Inc, Chugai Pharmaceutical Co, Ltd., Daiichi Sankyo Co., Ltd., Takeda Pharmaceutical Co., Ltd., AbbVie GK, Asahikasei Pharma Corp., Mitsubishi Tanabe Pharma Co., Pfizer Japan Inc., Eisai Co., Ltd., AYUMI Pharmaceutical Corporation, Nipponkayaku Co. Ltd., Novartis Pharma K.K., Grant/research support from: AbbVie, Asahi Kasei, Astellas, AstraZeneca, AYUMI, Bristol-Myers Squibb, Chugai, Daiichi Sankyo, Eisai, Eli Lilly Japan, Janssen, Mitsubishi Tanabe, Nippon Kayaku, Novartis, Pfizer Japan Inc, Taiho, Taisho Toyama, Takeda, Teijin, Grant/research support from: Astellas Pharma Inc., Bristol Myers Squibb, Chugai Pharmaceutical Co., Ltd., Mitsubishi Tanabe Pharma Co., Pfizer Japan Inc., Santen Pharmaceutical Co., Ltd. Takeda Pharmaceutical Co., Ltd., Teijin Pharma Ltd., AbbVie GK, Asahi 